\title{
A NOTE ON VECTOR-VALUED HARDY AND PALEY INEQUALITIES
}

\author{
OSCAR BLASCO
}

(Communicated by William J. Davis)

\begin{abstract}
The values of $p$ and $q$ for $L_{p}\left(L_{q}\right)$ that satisfy the extension of Paley and Hardy inequalities for vector-valued $H^{1}$ functions are characterized. In particular, it is shown that $L_{2}\left(L_{1}\right)$ is a Paley space that fails Hardy inequality.
\end{abstract}

\section{INTRODUCTION}

In [BP] the vector-valued analogue of two classical inequalities in the theory of Hardy spaces were investigated. A complex Banach space $X$ is said to be a Paley space if

$$
\left(\sum_{k=0}^{\infty}\left\|\hat{f}\left(2^{k}\right)\right\|^{2}\right)^{1 / 2} \leq C\|f\|_{1} \quad \text { for all } f \in H^{1}(X) .
$$

A complex Banach space $X$ is said to verify vector-valued Hardy inequality (for short $X$ is a (HI)-space) if

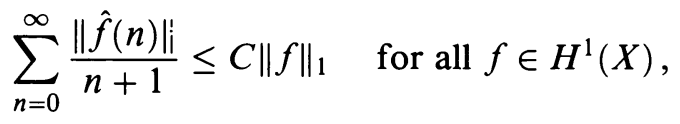

where $H^{1}(X)=\left\{f \in L^{1}(\mathbb{T}, X): \hat{f}(n)=0\right.$ for $\left.n<0\right\}$.

Both inequalities can be regarded in the framework of vector-valued extensions of multipliers from $H^{1}$ to $l^{1}$. Recall that a sequence $\left(m_{n}\right)$ is a $\left(H^{1}-l^{1}\right)$ multiplier, to be denoted by $m_{n} \in\left(H^{1}-l^{1}\right)$, if $T_{m_{n}}(f)=\left(\hat{f}(n) m_{n}\right)$ defines a bounded operator from $H^{1}$ into $l^{1}$.

The $\left(H^{1}-l^{1}\right)$-multipliers were characterized by $\mathrm{C}$. Fefferman in the following way (see [SW] for a proof):

$$
\left(H^{1}-l^{1}\right)=\left\{m_{n}: \sup _{s \geq 1}\left(\sum_{k \geq 1}\left(\sum_{j=k s+1}^{(k+1) s}\left|m_{j}\right|\right)^{2}\right)^{1 / 2}<\infty\right\} .
$$

Received by the editors December 26, 1990.

1991 Mathematics Subject Classification. Primary 42A45, 46E40, Secondary 42B30, 46B20.

Key words and phrases. Vector-valued Hardy spaces, Hardy inequality, Paley inequality.

The author was partially supported by the Grant C.A.I.C.Y.T. PS89-0106. 
A complex Banach space is said to have $\left(H^{1}-l^{1}\right)$-Fourier type if

(F) $\sum_{n=0}^{\infty}\|\hat{f}(n)\|\left|m_{n}\right| \leq C\|f\|_{1} \quad$ for all $f \in H^{1}(X)$ and for all $m_{n} \in\left(H^{1}-l^{1}\right)$.

The reader is referred to [BP] for examples of spaces having and failing these properties and for their connection with the notions of Rademacher type and Fourier type.

Using $(*)$ it is easy to see that any space of $\left(H^{1}-l^{1}\right)$-Fourier type must be a Paley and a (HI)-space. Unfortunately the only examples of spaces without $\left(H^{1}-l^{1}\right)$-Fourier type that we had at our disposal also behave badly with respect to the other two properties. The problem of finding a Paley space failing Hardy inequality or without $\left(H^{1}-l^{1}\right)$-Fourier type was left open (see [BP, Remark 4.1]).

Surprisingly it is enough to deal with Lebesgue spaces of mixed norm, namely, $L_{p}\left(L_{q}\right)$, to produce a simple example of Paley space failing Hardy inequality. In fact we shall see that $L_{2}\left(L_{1}\right)$ is such an example.

Given $1 \leq p \leq \infty,(\Omega, \Sigma, \mu)$ a $\sigma$-finite measure space, and a Banach space $Y$ we denote by $L_{p}(\mu, Y)$ the space of $Y$-valued strongly measurable functions such that $\|f\| \in L_{p}(\mu)$.

Throughout the paper $1 \leq p, q \leq \infty$ and we shall use the notation $L_{p}\left(L_{q}\right)=$ $L_{p}\left(\mathbb{T}, L_{q}(\mathbb{T})\right)$.

\section{PALEy SPACES}

For self-containedness of the paper, we provide here simple direct proofs of special cases of Corollary 3.2 and Theorem 3.2 of [BP] that show how the Paley property behaves with respect to the vector-valued extension.

Lemma 1. Let $1 \leq p \leq 2,(\Omega, \Sigma, \mu)$ be a $\sigma$-finite measure space and $Y$ a Paley space. Then $L_{p}(\mu, Y)$ is a Paley space.

Proof. The case $p=2$ is a simple consequence of Fubini's theorem. Let us assume $1 \leq p<2$ and $q=(2 / p)^{\prime}=2 /(2-p)$. Let us take $f(t)=\sum_{n \geq 0} x_{n} e f$ where $x_{n} \in L_{p}(\mu, Y)$.

$$
\begin{gathered}
\left(\sum_{k \geq 0}\left\|x_{2^{k}}\right\|_{L_{p}(\mu, Y)}^{2}\right)^{1 / 2}=\left(\sum_{k \geq 0}\left(\int_{\Omega}\left\|x_{2^{k}}(w)\right\|_{Y}^{p} d \mu(w)\right)^{2 / p}\right)^{1 / 2} \\
=\sup _{\sum \alpha_{k}^{q}=1}\left(\sum_{k \geq 0} \int_{\Omega}\left\|x_{2^{k}}(w)\right\|_{Y}^{p} \alpha_{k} d \mu(w)\right)^{1 / p} \\
\leq\left(\int_{\Omega}\left(\sum_{k \geq 0}\left\|x_{2^{k}}(w)\right\|_{Y}^{2}\right)^{p / 2} d \mu(w)\right)^{1 / p} \\
\leq C\left(\int_{\Omega}\left(\frac{1}{2 \pi} \int_{-\pi}^{\pi} \|\left.\sum_{n \geq 0} x_{n}(w) e^{\mathrm{int}}\right|_{Y} d t\right)^{p} d \mu(w)\right)^{1 / p}
\end{gathered}
$$




$$
\begin{aligned}
& =C \sup _{\|h\|_{p^{\prime}}=1} \int_{\Omega} \int_{-\pi}^{\pi}\left\|\sum_{n \geq 0} x_{n}(w) e^{\mathrm{int}}\right\|_{Y} h(w) d t d \mu(w) \\
& \leq C \int_{-\pi}^{\pi}\left\|\sum_{n \geq 0} x_{n}(w) e^{\mathrm{int}}\right\|_{L_{p}(\mu, Y)} d t=C \int_{-\pi}^{\pi}\|f(t)\|_{L_{p(\mu, Y)}} d t .
\end{aligned}
$$

Theorem 1. $L_{p}\left(L_{q}\right)$ is a Paley space if and only if $1 \leq p, q \leq 2$.

Proof. It is clear from the definition that a Paley space must have cotype 2 . (Recall that the notion of cotype can be defined with $e^{i 2^{k} t}$ instead of Rademacher functions.) Now the cotype 2 condition forces the values of $1 \leq p, q \leq 2$.

To get the converse, observe that the classical Paley inequality together with Lemma 1 for $Y=\mathbb{C}$ gives that $L_{q}$ is a Paley space for $1 \leq q \leq 2$. Now apply Lemma 1 again.

\section{(HI)-SPACES}

Let us now prove the main result of the paper.

Theorem 2. If $1<p \leq \infty$ then $l_{p}\left(H^{1}\right)$ is not a (HI)-space.

Proof. The case $p=\infty$ is immediate since it does contain $c_{0}$ and $c_{0}$ fails Hardy (see $[\mathrm{BP}]$ ). We assume then $1<p<\infty$. Let us consider the function

$$
f(z)=\frac{1}{(1-z)^{p}} \frac{z}{\log (1-z)^{-1}}=\sum_{n=0} a_{n} z^{n} .
$$

Let us recall the following estimates (see [ $\mathrm{L}$ p. 93-96]

$$
a_{n} \sim \frac{n^{p-1}}{\log n} \quad(n \rightarrow \infty)
$$

$$
M_{1}(f, r) \sim \frac{(1-r)^{1-p}}{\log (1-r)^{-1}} \quad(r \rightarrow 1),
$$

where $M_{1}(f, r)=\frac{1}{2 \pi} \int_{-\pi}^{\pi}\left|f\left(r e^{i t}\right)\right| d t$. by

Let us define $\Phi(z)=\sum_{n=0}^{\infty} x_{n} z^{n}$ for $|z|<1$ where $x_{n} \in l_{p}\left(H^{1}\right)$ are given

$$
x_{n}(k, w)=\left(2^{k(1-p)} a_{n}\left(1-1 / 2^{k}\right)^{n} w^{n}\right) \quad(k \in \mathbb{N},|w|=1) .
$$

Note that $\left\|x_{n}\right\|_{l_{p}\left(H^{\prime}\right)} \leq C a_{n}$ shows that $\Phi$ is analytic on the open unit disc and takes values in $l_{p}\left(H^{1}\right)$.

Let us define $F(z)=\lim _{r \rightarrow 1} \Phi(r z)$ for $|z|=1$ if the radial limit exists. We shall show that $F \in H^{1}\left(l_{p}\left(H^{1}\right)\right)$.

$$
\Phi(z)(k, w)=2^{k(1-p)} \sum_{n=0}^{\infty} a_{n}\left(1-\frac{1}{2^{k}}\right)^{n} w^{n} z^{n}=2^{k(1-p)} f\left(\left(1-\frac{1}{2^{k}}\right) w z\right) .
$$

Using (2) we have

$$
\|\Phi(z)\|_{l_{p}\left(H^{1}\right)}=\left(\sum_{k=1}^{\infty} 2^{k p(1-p)} M_{1}^{p}\left(f,\left(1-2^{-k}\right)|z|\right)\right)^{1 / p} \leq C\left(\sum_{k=1}^{\infty} \frac{1}{k^{p}}\right)^{1 / p}<\infty .
$$


Now since $\Phi$ is uniformly bounded then $\sup _{0 \leq r<1} M_{1}(\|\Phi\|, r)<\infty$. Using the fact that for $1 \leq p<\infty$ the Banach space $l_{p}\left(H^{1}\right)$ is a separable dual by a routine argument, we show that the radial limit $F(z)$ exists almost everywhere and that $F \in H^{1}\left(l_{p}\left(H^{1}\right)\right)$ for $1<p<\infty$.

On the other hand

$$
\left\|x_{n}\right\|_{l_{p}\left(H^{1}\right)}=a_{n}\left(\sum_{k=1}^{\infty} 2^{k p(1-p)}\left(1-\frac{1}{2^{k}}\right)^{n p}\right)^{1 / p} \geq a_{n}\left(\sum_{k \geq \log _{2} n}^{\infty} 2^{k p(1-p)}\left(1-\frac{1}{n}\right)^{n p}\right)^{1 / p}
$$

Since $(1-1 / n)^{n p}$ converges to $e^{-p}$, for $n$ big enough we have

$$
\left\|x_{n}\right\|_{l_{p}\left(H^{1}\right)} \geq C_{p} a_{n}\left(\sum_{k \geq \log _{2} n}^{\infty} 2^{k p(1-p)}\right)^{1 / p} \geq C_{p} a_{n} n^{1-p} .
$$

Now using estimate (1) we get $\sum_{n=1}^{\infty}\left\|x_{n}\right\|_{l_{F}\left(H^{1}\right)} /(n+1)=\infty$.

Remark. If $1<p<\infty$ then $l_{p}\left(H_{1}\right)$ is a Paley space but is not a (HI)-space. (Hence it does not have $\left(H^{1}-l^{q}\right)$-Fourier type.)

Theorem 3. $L_{p}\left(L_{q}\right)$ is a (HI)-space if and only if either $1<p, q<\infty$ or $p=1$ and $1 \leq q<\infty$.

Proof. Let us first show that under such assumptions on $p, q$ we get (HI)spaces. It is an application of Fubini's theorem that if $Y$ is a (HI)-space then $L_{1}(\mu, Y)$ is a $(\mathrm{HI})$-space. Combining this with the result that every $B$-convex space (Rademacher type bigger than 1 ) is a (HI)-space (see [BP, Bo]) we get this implication.

For the other implication observe that the cases $p=\infty$ or $q=\infty$ must be excluded because then $L_{p}\left(L_{q}\right)$ would contain $c_{0}$. The case $q=1$ follows from Theorem 1, since $l_{p}$ embbeds into $L_{p}(\mathbb{T})$ and $H^{1}$ into $L_{1}(\mathbb{T})$.

\section{ACKNOWLEDGMENT}

I am very grateful to A. Pelczynski for helpful conversations on the subject and to $\mathrm{B}$. Koremblum for his comments on related problems that inspired me the proof of Theorem 2 . I would like also to thank the referee for his suggestions.

\section{REFERENCES}

[BP] O. Blasco, and A. Pelczynski, Theorems of Hardy and Paley for vector-valued analytic functions and related classes of Banach spaces, Trans. Amer. Math. Soc. 323 (1991), 335-367.

[Bo] J. Bourgain, Vector-valued Hausdorff-Young inequality and applications, Geometric Aspects in Functional Analysis, Israel Seminar (GAFA) 1986-87, Lecture notes in Math, vol. 1317, Springer-Verlag, pp. 239-249 Berlin, 1988.

[D] P. Duren, Theory of $H_{p}$-spaces, Academic Press, New York, 1970.

[L] J. E. Littlewood, Lectures on the theory of functions, Oxford Univ. Press, London, 1944.

[SW] S. J. Szarek, and T. Wolniewicz, A proof of Fefferman's theorem on multipliers, Inst. Math. Polish Acad. Sci., preprint 209, Warzawa, 1980. 